\title{
Prevalence of Malnutrition and Associated Factors among Children in Rural Ethiopia
}

\author{
Neima Endris, Henok Asefa, and Lamessa Dube \\ Department of Epidemiology, Jimma University, Jimma, Ethiopia \\ Correspondence should be addressed to Lamessa Dube; dubelamessa@yahoo.com
}

Received 1 March 2017; Accepted 26 April 2017; Published 17 May 2017

Academic Editor: Apollinaire G. Horo

Copyright (C) 2017 Neima Endris et al. This is an open access article distributed under the Creative Commons Attribution License, which permits unrestricted use, distribution, and reproduction in any medium, provided the original work is properly cited.

\begin{abstract}
Background. Child malnutrition continues to be the leading public health problem in developing countries. In Ethiopia, malnutrition is a leading cause of child illness and death. Recently the composite index of anthropometric failure (CIAF) has been implemented to measure the prevalence of malnutrition. This index presents a more complete picture compared with the previous conventional indices. In this study, CIAF was used to determine the prevalence of malnutrition among children aged 0-59 months in rural Ethiopia. Methods. Data was extracted from the 2014 Ethiopian Mini Demographic and Health Survey (EMDHS) for this study. A total of 3095 children were included in the analysis. The composite index of anthropometric failure (CIAF) was used to measure the nutritional status of the children. Logistic regression was fitted, to identify factors associated with malnutrition among children in rural Ethiopia, using STATA 13. Result. The prevalence of malnutrition among rural children in Ethiopia was $48.5 \%$. Age of the children, preceding birth interval, educated status of mother, wealth status, and region were factors independently associated with nutritional status of children in rural Ethiopia. Conclusion. The prevalence of malnutrition among children in rural Ethiopia was high. A child older than 12 months, having uneducated mother, living in a household with poor wealth status, born with short birth interval, and living in some region of the country are associated with increased odds of being malnourished.
\end{abstract}

\section{Introduction}

Child malnutrition continues to be the leading public health problem in developing countries. Globally, there were 165 million stunted, 99 million underweight, and 51 million wasting children by year 2012. It kills 3.1 million under-five children every year [1]. Under-five children are the most susceptible age group for malnutrition. Malnutrition at the early stages of life can increase risk infections, morbidity, and mortality together with decreased mental and cognitive development. The effect of child malnutrition is long lasting and goes beyond childhood. For instance, malnutrition during early age decreases the educational achievement and labor productivity and raises the risk of chronic illnesses in later age [1-3].

Malnutrition is the major cause of illness and death among under-five children in Ethiopia. The rate of malnutrition among under-five children in the country is among the highest in the world and Sub-Saharan Africa. Moreover, malnutrition is the underlying cause for three-fifth of child death in the country [4-6]. According to the 2014 Ethiopian Mini Demographic and Health Survey (EMDHS) report, 42\%, $26.7 \%$, and $9 \%$ of under-five children were stunted, underweight, and wasted, respectively. The problem is even worse in rural areas. For instance, the prevalence of underweight and stunting among rural children was $27 \%$ and $42 \%$ compared with only $13 \%$ and $24 \%$ among urban children, respectively [7].

The planning of an appropriate intervention requires the knowledge of the extent and the underlying causes of the problem. To this end, very few studies have been conducted regarding childhood malnutrition in rural Ethiopia. All of them were small scale surveys limited in particular regions of the country [4, 6, 8-10]. Hence, they did not provide a full picture of the extent of the problem on country level. Moreover, these studies used the conventional indicators of nutritional status to measure the prevalence of malnutrition in under-five children. However, a number of studies pointed out that the use of conventional indicators provides only the categorization of children into the general categories of 
malnutrition and does not determine the overall prevalence of malnutrition associated with multiple failures. Consequently, these indicators underestimate the prevalence of malnutrition due to the potential overlap of children into multiple categories of anthropometric failure [11-16].

Therefore, in this study we used a recently developed and relatively robust alternative indicator of malnutrition-the composite index of anthropometric failure (CIAF) to determine the prevalence of malnutrition and associated factors among under-five children in rural Ethiopia.

\section{Methods}

2.1. Data Source. For this study, we used the 2014 Mini Ethiopian Demographic and Health Survey data. A nationwide survey was conducted by the Ethiopian Central Statistical Agency (ECSA) under the aegis of the Ministry of Health for four months from January to April 2014. A total of 8,475 households 2,556 from urban and 5,919 from rural areas were covered. A two-stage stratified-cluster sampling design was used to select these households [7].

Then data was extracted, on children aged 0-59 months, from this survey data to determine the prevalence of malnutrition and the associated factors among rural children in Ethiopia using CIAF as a measure of nutritional status of the children. In household where there were more than one under-five child, data of only the younger one was extracted.

2.2. Measurements. The outcome variable of interest in this study was nutritional status among under-five children measured using CIAF. Children are categorized into seven groups, namely, no failure; wasting only; wasting and underweight; wasting, stunting, and underweight; stunting and underweight; stunting only; and underweight only. A child who did not suffer from any anthropometric failure was classified as "no failure." A child with acceptable Weight-for-Age (WA) and Height-for-Age (HA) but who had subnormal Weightfor-Height (WH) (that is, below $-2 \mathrm{z}$-scores of the WHO 2006 standards) was classified as "wasting only." A child with acceptable WA and HA but who had subnormal WH (that is, below -2 z-scores of the WHO 2006 standards) was classified as "wasting only." A child with acceptable HA but low WA and WH (that is, below -2 z-scores of the WHO 2006 standards) was classified "wasting and underweight." A child who suffered from anthropometric failure on all three measures was "wasting, stunting and underweight." A child with low WA and HA (that is, below -2 z-scores of the WHO 2006 standards) but acceptable WH was classified as "stunting and underweight." A child with low HA (that is, below -2 z-scores of the WHO 2006 standards) but acceptable WA and WH was categorized as "stunting only." A child with low WA only (that is, below -2 z-scores of the WHO 2006 standards) was categorized as "underweight only." Thus a child is regarded as malnourished, as measured in CIAF, if s/he is suffering from any anthropometric failure above $[11,14,15]$.

A range of information was extracted from the Mini EDHS including (i) sociodemographic and economic characteristics (child's age, child's sex, birth order, preceding birth interval, ANC visit, maternal marital status, maternal educational status, maternal age at first birth, size of household, head of household, region of residence, and wealth index). The preceding birth interval was computed from the data collected by questions "Now I would like to record birth date of all your births, whether still alive or not, starting with the first one you had." Except for the first born child, the preceding birth interval was computed by calculating the deference between the last and the preceding birth dates. The current marital status was categorized into currently in union or not in union. Legal or formal married women and an informal union in which a man and a woman live together were categorized as currently in union, otherwise not in union. ANC visit was measured by directing "For last birth, did you see anyone for antenatal care for during pregnancy?" a yes or no question.

The household wealth index is a socioeconomic index constructed as an indicator of the level of wealth that is consistent with expenditure and income measures. In the EDHS the index is based on data from household ownership of assets and consumer goods such as source of drinking water, type of toilet facilities, type of fuel, ownership of various durable goods, and other characteristics relating to socioeconomic status of the household. A factor score generated through principal components analysis was assigned to each asset, and the resulting asset scores were standardized in relation to a normal distribution [7]. For this analysis, the wealth index was grouped into three categories: poor, middle, and rich.

2.3. Data Analysis. Data were extracted from EDHS 2014 database, exported, edited, cleaned, coded, and analyzed using STATA version 13 for Windows. Then a weighted analysis was conducted using the same sampling weight given for each region by EDHS to make the data nationally representative [7]. First, descriptive analysis was done. Then bivariate analysis was done to identify factors associated with malnutrition. Those variables in the bivariate analysis with $P$ value less than 0.25 were considered as candidates to be included in the multivariable logistic regression model. The multivariable logistic regression was performed by the backward stepwise variable selection method with probability of removal 0.10. Finally, the adequacy of the model was checked by using Hosmer and Lemeshow goodness-of-fit test.

\section{Results}

3.1. Socioeconomic and Demographic Characteristics of Study Participants. A total of 3095 weighted children living in rural area were included in the analysis among which $50.1 \%$ were females. The mean age of the children was $26.4 \pm 16.2$ months. Mean age of the mother at first birth was $18.7 \pm 3.4$ years. Majority of mothers (93\%) were in union by marital status. Almost $87 \%$ of the children had preceding birth interval greater than twelve months. More than half (56.5\%) of the children were living in a household with more than five members. Majority of the mothers $(67.3 \%)$ had no education. Most of the households (89.8\%) were male headed and 50.5\% were found in the poor wealth quintile (Table 1). 
TABLE 1: Demographic and socioeconomic characteristics of children aged 0-59 months, rural Ethiopia, 2014.

\begin{tabular}{|c|c|}
\hline Characteristics & Frequency (\%) \\
\hline \multicolumn{2}{|l|}{ Sex of the child } \\
\hline Male & 1545 (49.9) \\
\hline Female & $1550(50.1)$ \\
\hline \multicolumn{2}{|l|}{ Age of child (in months) } \\
\hline$<6$ & $346.6(11.2)$ \\
\hline $6-11$ & $356.3(11.5)$ \\
\hline $12-23$ & $694.1(22.4)$ \\
\hline $24-35$ & $687.9(22.2)$ \\
\hline $36-47$ & $592.1(19.1)$ \\
\hline $48-59$ & $417.7(13.5)$ \\
\hline \multicolumn{2}{|l|}{ Birth order } \\
\hline 1 & $405.8(14.4)$ \\
\hline $2-3$ & $862.1(30.6)$ \\
\hline $4-5$ & $758.9(27.0)$ \\
\hline $6^{+}$ & $793.5(28.1)$ \\
\hline \multicolumn{2}{|l|}{ Preceding birth interval } \\
\hline$\leq 24$ months & $406(13.1)$ \\
\hline$>24$ months & $2689(86.9)$ \\
\hline \multicolumn{2}{|l|}{ Current marital status } \\
\hline Currently not in union & $116(7.0)$ \\
\hline Currently in union & $1550(93.0)$ \\
\hline \multicolumn{2}{|l|}{ Mother age at first birth } \\
\hline 18 and less & $917.2(55.6)$ \\
\hline 19 and above & $734(44.4)$ \\
\hline \multicolumn{2}{|l|}{ Household family size } \\
\hline Five and less & $1346(43.5)$ \\
\hline Six and above & $1749(56.5)$ \\
\hline \multicolumn{2}{|l|}{ Region of residence } \\
\hline Oromiya & $1264(40.9)$ \\
\hline Amhara & $754.6(24.4)$ \\
\hline SNNP & $741.3(23.9)$ \\
\hline Tigray & $198(6.4)$ \\
\hline Somalia & $55.1(1.8)$ \\
\hline Benshangul-Gumuz & $31.2(1.0)$ \\
\hline Affar & $26.2(0.8)$ \\
\hline Gambela & $12.4(0.4)$ \\
\hline Dire Dawa & $7.2(0.2)$ \\
\hline Harari & $4.6(0.15)$ \\
\hline \multicolumn{2}{|l|}{ Mother's education } \\
\hline No education & $1898(67.3)$ \\
\hline Primary education & $848.8(30.1)$ \\
\hline Secondary and above & $73.2(2.6)$ \\
\hline \multicolumn{2}{|l|}{ Sex of household head } \\
\hline Male & $2778(89.8)$ \\
\hline Female & $316.3(10.2)$ \\
\hline Two and less & $2797(90.4)$ \\
\hline More than two & $298(9.6)$ \\
\hline
\end{tabular}

TABLE 1: Continued.

\begin{tabular}{lc}
\hline Characteristics & Frequency (\%) \\
\hline Wealth status & \\
Poor & $1562(50.5)$ \\
Medium & $717.4(23.2)$ \\
Rich & $815.2(26.3)$ \\
\hline ANC visit & \\
Yes & $1250(55.7)$ \\
No & $994(44.3)$ \\
\hline
\end{tabular}

TABLE 2: Prevalence of malnutrition based on CIAF among underfive-year-old children in rural Ethiopia, 2014.

\begin{tabular}{lcc}
\hline Group & Description of the group & Frequency (percentage) \\
\hline I & No failure & $1594(51.5 \%)$ \\
II & Wasting only & $101.4(3.3 \%)$ \\
III & Wasting and underweight & $85.3(2.8 \%)$ \\
IV & Wasting, stunting, and underweight & $113(3.7 \%)$ \\
V & Stunting and underweight & $599.2(19.4 \%)$ \\
VI & Stunting only & $563(18.2 \%)$ \\
VII & Underweight only & $39(1.3 \%)$ \\
\hline Total & & $3094.9(100 \%)$ \\
\hline CIAF (II + III + IV + V + VI + VII $)=48.5 \%$. &
\end{tabular}

3.2. Prevalence of Malnutrition. Based on CIAF, $48.5 \%$ of children living in rural Ethiopia were malnourished. From this, $22.7 \%$ had single anthropometric failure and $25.9 \%$ had multiple anthropometric failures. The prevalences of the seven groups of nutritional status were presented in Table 2. Malnutrition among rural children in Ethiopia using the conventional measures was found to be underweight $27 \%$, wasting $9.7 \%$, and stunting $41.2 \%$.

3.3. Factors Associated with Nutritional Status of Children. The result of multivariable logistic regression analysis showed that age of the child, preceding birth interval, region of residence, maternal education, and economic status of the household were factors independently associated with nutritional status of children.

Compared to children less than six months old, the odds of malnutrition among children in the age group of $12-23$ months were 2.6 times higher $[\mathrm{AOR}=2.63,95 \% \mathrm{CI}$ : $1.72-4.01$ ], in the age group of 24-35 months were 4 times higher [AOR $=3.97,95 \% \mathrm{CI}: 2.52-6.26]$, in the age group of $36-47$ months were 3.5 times higher [AOR $=3.51$, 95\% CI: 2.32-5.31], and in the age group of 48-59 months were 2.75 times higher $[\mathrm{AOR}=2.75,95 \% \mathrm{CI}: 1.64-4.63]$.

Children whose preceding birth interval was less than two years were 1.43 times at higher risk of being malnourished compared to children with preceding birth interval greater than 24 months [adjusted odds ratio (AOR) $=1.43,95 \%$ CI: 1.02-2.04]. The risk of being malnourished among children whose mother did not attend education was 1.32 times higher compared to children whose mothers attended primary education $[\mathrm{AOR}=1.32$, 95\% CI: $1.02-1.72]$.

Compared to children residing in households with poor economic status, the probability of being malnutrition among 
TABLE 3: Factors independently associated with nutritional status of rural children in Ethiopia, 2014.

\begin{tabular}{|c|c|c|}
\hline Variables & Adjusted OR & $95 \% \mathrm{CI}$ \\
\hline \multicolumn{3}{|l|}{ Age of child (in months) } \\
\hline$<6$ & 1 & \\
\hline $6-11$ & 0.78 & $0.48-1.24$ \\
\hline $12-23$ & 2.63 & $1.72-4.01^{*}$ \\
\hline $24-35$ & 3.97 & $2.52-6.26^{*}$ \\
\hline $36-47$ & 3.51 & $2.32-5.31^{*}$ \\
\hline $48-59$ & 2.75 & $1.64-4.63^{*}$ \\
\hline \multicolumn{3}{|l|}{ Preceding birth interval } \\
\hline$>24$ months & 1 & \\
\hline$\leq 24$ months & 1.43 & $1.023-2.04^{*}$ \\
\hline \multicolumn{3}{|l|}{ Mother's educational status } \\
\hline Primary & 1 & \\
\hline No education & 1.32 & $1.02-1.72^{*}$ \\
\hline Secondary and above & 0.49 & $0.19-1.29$ \\
\hline \multicolumn{3}{|l|}{ Region of residence } \\
\hline Tigray & 1 & \\
\hline Afar & 1.12 & $0.62-2.06$ \\
\hline Amhara & 0.71 & $0.44-1.15$ \\
\hline Oromiya & 0.62 & $0.38-1.00$ \\
\hline Somali & 0.79 & $0.46-1.37$ \\
\hline Benshangul-Gumuz & 0.70 & $0.37-1.32$ \\
\hline SNNP & 0.59 & $0.37-0.98^{*}$ \\
\hline Gambella & 0.47 & $0.26-0.82^{*}$ \\
\hline Harari & 0.59 & $0.36-0.97^{*}$ \\
\hline Dire Dawa & 0.78 & $0.43-1.42$ \\
\hline \multicolumn{3}{|l|}{ Wealth status } \\
\hline Poor & 1 & \\
\hline Medium & 0.66 & $0.45-0.95^{*}$ \\
\hline Rich & 0.63 & $0.62-0.65^{*}$ \\
\hline
\end{tabular}

Note. ${ }^{*}$ Significant at $P$ value of 0.05 ; SNNP: Southern Nations Nationalities and Peoples.

children residing in households with medium and rich economic status decreased by $34 \%$ and $37 \%$, respectively. This study also showed that when compared to children in Tigray region, the risk of malnutrition was decreased by $55 \%$ [AOR $=0.47,95 \%$ CI: $0.26-0.82], 41 \%[\mathrm{AOR}=0.59,95 \%$ CI: $0.37-0.98]$, and $41 \%$ [AOR $=0.59,95 \%$ CI: $0.36-0.97]$ among children living in Gambela, Southern Nations Nationalities and Peoples (SNNP), and Harari regions, respectively (Table 3).

\section{Discussion}

The findings of this study revealed that almost half of children aged 0-59 months were malnourished and the risk factors were region of residence, education of mother, economic status, age of child, and preceding birth interval.

The prevalence of malnutrition among rural children was $48.5 \%$ based on CIAF measurement. This prevalence is higher compared with the results of the Ethiopian Demographic and Health Survey and regional as well as national levels studies $[4,7,17]$. This difference might be due to the difference in the methods used to assess nutritional status. A number of literatures reported that the conventional methods used to assess malnutrition in a population underestimate the prevalence of malnutrition compared with CIAF [14, 15]. Estimated CIAF of African countries based on 2008 data ranges from $35.6 \%$ in Ghana to $51.6 \%$ [16]. Compared to Asian countries like China and India where reported CIAF are $21.7 \%$ and $32.7 \%$, respectively $[14,15]$, the result of this study is higher.

In this study, age of the child was found to be significantly associated with nutritional status, as the age of child increases the risk of being malnourished increases. This finding is in line with studies done in Tigray region, Nigeria, and Bangladesh $[6,18,19]$. One conceivable clarification could be because of the late introduction of supplementary food with low nutritional quality [14]. The other reason might be that a large portion of guardians in rural areas are neglecting to fulfill optimal food requirements of their children as the child's age increases [20].

Preceding birth interval is the other important variable which is associated with nutritional status of children. In particular, there is an inverse relationship between the length of the preceding birth interval and the proportion of children who are malnourished. This finding is also consistent with the report of Ethiopian DHS 2011 and 2014 and other studies conducted in Ethiopia, Nigeria, India, and Bangladesh [4, 7, 18-21]. For the newborns, the larger birth interval results into better care and more time allocation for the nutrition and wellbeing [20].

This study demonstrated that there is significant association between maternal education and nutritional status of children. This finding is consistent with other studies done in Ethiopia, Bangladesh, and Nigeria $[8,14,18]$. This is because educated mothers are more conscious about their children's health; and they tend to look after their children in a better way [18].

This study also indicated that there is association between region of residence and nutritional status. Earlier survey has also shown a very low prevalence of malnutrition in these regions [22]. The watched distinction may reflect natural imperatives, more awful broad living conditions, harmful sociocultural practices, unequal intrahousehold food distribution, seasonal food insecurity, poor public facilities, and other related factors [21].

In addition, our study showed that under-five children from poor wealth status are at a higher risk of malnutrition compared with children from rich households. This is consistent with other studies done in Ethiopia, Bangladesh, Nigeria, and India $[4,14,18,23]$.

Finally, this study is limited in that it does not take into account some important variables that affect the nutritional status of children such as dietary aspects.

\section{Conclusions}

In conclusion, prevalence of malnutrition among under-five children in rural Ethiopia was high. Malnutrition continued 
to be a substantial burden in the country. And age of the child, maternal education, wealth status, and birth interval were associated with nutritional status of children in rural Ethiopia.

Special attention needs to be given to the problem of malnutrition among rural children in the country since the problem is extensive compared to previous studies done using the conventional methods of assessing nutritional status. We recommend policy makers to use CIAF as a measure of nutritional status in order to estimate the overall burden of malnutrition in the population.

\section{Abbreviations}

ANC: Antenatal care

AOR: Adjusted odds ratio

CIAF: Composite index of anthropometric

failure

CSA: Central Statistical Agency

EMDHS: Ethiopian Mini Demographic and Health Survey

SNNPS: South Nations Nationalities and Peoples Regional State.

\section{Additional Points}

Availability of Data and Material. The dataset supporting the conclusions of this study is not available because this study is based on national data and we have no mandate to share the dataset.

\section{Ethical Approval}

Ethical clearance was obtained from Ethical Review Committee of College of Health Science, Jimma University. Additionally, official permission was secured from the Central Statistical Agency to use the DHS dataset for this study.

\section{Consent}

Permission to disseminate the study finding through publication was obtained from CSA.

\section{Disclosure}

This research received no specific grant from any funding agency in the public, commercial, or not-for-profit sectors.

\section{Conflicts of Interest}

No conflicts of interest prevail among researchers.

\section{Authors' Contributions}

Neima Endris participated in the design of the study and data extraction, performed statistical analysis, and drafted the first manuscript. Henok Asefa and Lamessa Dube participated in the design of the study and data extraction, performed statistical analysis, and reviewed the manuscript. All authors have read and approved the final manuscript.

\section{Acknowledgments}

The authors acknowledge CSA for granting access to the dataset.

\section{References}

[1] R. E. Black, C. G. Victora, S. P. Walker et al., "Maternal and child undernutrition and overweight in low-income and middleincome countries," The Lancet, vol. 382, no. 9890, pp. 427-451, 2013.

[2] "A life free from hunger: Tackling Child Malnutrition: report by Save the children International," Geneva. Feb, 2012.

[3] World Health Organization, Essential Nutrition Actions: Improving Maternal, Newborn, Infant and Young Child Health and Nutrition, WHO Document Publications Services, Geneva, Switzerland, 2013.

[4] D. Headey, "An analysis of trends and determinants of child under nutrition in Ethiopia, 2001-2011," International Food Policy Research Institute Working Pape 70, EDRI, Washington Dc, USA.

[5] Ethiopia National Nutrition Programme Implementing Sectors Declaration, "Government of the Federal Democratic Republic of Ethiopia (2013-2015)”.

[6] Save the children UK Ethiopia, National Nutrition Strategy: Review and Analysis of Progress and Gaps, One Year on, Save the children UK, London, UK, 2009.

[7] Central Statistical Agency, Ed., Ethiopia Mini Demographic and Health Survey 2014, CSA, Addis Ababa, Ethiopia, 2014.

[8] K. Mengistu, K. Alemu, and B. Destaw, "Prevalence of malnutrition and associated factors among children aged 6-59 months at hidabu abote district, north shewa, Oromia regional state," Journal of Nutritional Disorders \& Therapy, vol. T1, article 001, 2013.

[9] B. M. Yalew, "Prevalence of malnutrition and associated factors among children age 6-59 months at lalibela town administration, North WolloZone, Anrs, Northern Ethiopia," Journal of Nutritional Disorders \& Therapy, vol. 4, no. 1, p. 132, 2014.

[10] M. Tesfaye, "Bayesian approach to identify predictors of children Nutritional status in Ethiopia," 2009.

[11] S. Nandy, M. Irving, D. Gordon, S. V. Subramanian, and G. D. Smith, "Poverty, child undernutrition and morbidity: new evidence from India," Bulletin of the World Health Organization, vol. 83, no. 3, pp. 210-216, 2005.

[12] P. Svedberg, Poverty and under Nutrition: Theory, Measurement and Policy, Oxford India Paperbacks, New Delhi, India, 2000.

[13] M. R. Berger, C. Fields-Gardner, A. Wagle, and C. B. Hollenbeck, "Prevalence of malnutrition in human immunodeficiency virus/acquired immunodeficiency syndrome orphans in the nyanza province of kenya: a comparison of conventional indexes with a composite index of anthropometric failure," Journal of the American Dietetic Association, vol. 108, no. 6, pp. 10141017, 2008.

[14] A. Dasgupta, R. Parthasarathi, V. Ram Prabhakar, R. Biswas, and A. Geethanjali, "Assessment of under nutrition with composite index of anthropometric failure (CIAF) among under-five children in a rural area of west bengal," Indian Journal of Community Health, vol. 26, no. 2, pp. 132-138, 2014. 
[15] L. Pei, L. Ren, and H. Yan, "A survey of undernutrition in children under three years of age in rural Western China," BMC Public Health, vol. 14, no. 1, article 121, 2014.

[16] S. Nandy and P. Svedberg, "Handbook of Anthropometry," in The Composite Index of Anthropometric Failure (CIAF): An Alternative Indicator for Malnutrition in Young Children, pp. 127-137, Springer New York, New York, NY, USA, 2014, https:// link.springer.com/chapter/10.1007\%2F978-1-4419-1788-1_6.

[17] B. Teshome, W. Kogi-Makau, Z. Getahun, and G. Taye, "Magnitude and determinants of stunting in children underfive years of age in food surplus region of Ethiopia: the case of West Gojam Zone," Ethiopian Journal of Health Development, vol. 23, no. 2, pp. 98-106, 2006.

[18] I. O. Sebanjo, O. O. Adeodu, and E. A. Adejuyigbe, Influence of socio-economic factors on nutritional status of children in a rural community of Osun State, Nigeria, 2009.

[19] K. S. Mostafa, "Socio-economic determinants of severe and moderate stunting among under-five children of rural Bangladesh," Malaysian Journal of Nutrition, vol. 17, pp. 105-118, 2011.

[20] R. E. A. Khan and M. A. Raza, "Determinants of malnutrition in Indian children: new evidence from IDHS through CIAF," International Journal of Methodology, vol. 50, no. 1, pp. 299-316, 2016.

[21] G. Woldemariam and T. Genebo, Determinants of Nutritional Status of Women and Children in Ethiopia, ORC Macro, Calverton, Md, USA, 2002.

[22] T. Sebsibe and A. Yinges, "Urban-rural differentials in child undernutrition in Ethiopia," International Journal of Nutrition and Metabolism, vol. 7, no. 1, pp. 15-23, 2015.

[23] A. Shahjada, B. K. Sharma, S. Sharma, P. Mahashabde, and A. Bachhotiya, "Effects of birth interval on nutritional status of under five children in peri-urban area of Madhya Pradesh, India," International Journal of Medical Science and Public Health, vol. 3, no. 6, p. 723, 2014. 


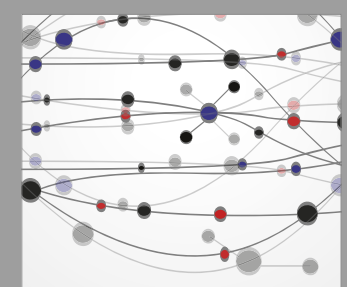

The Scientific World Journal
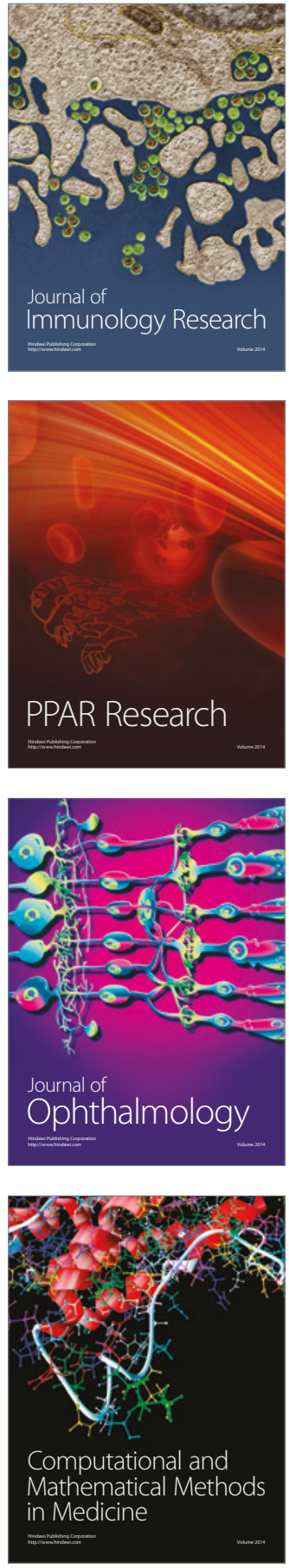

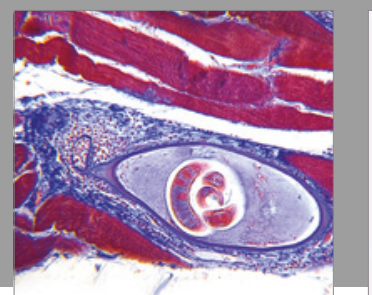

Gastroenterology Research and Practice
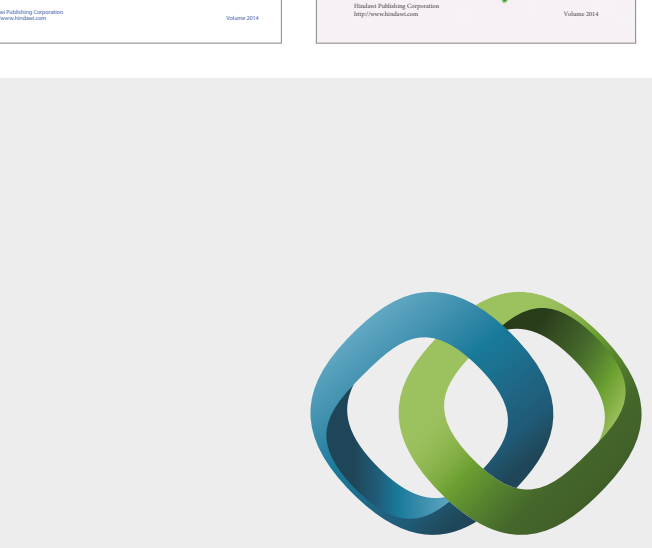

\section{Hindawi}

Submit your manuscripts at

https://www.hindawi.com
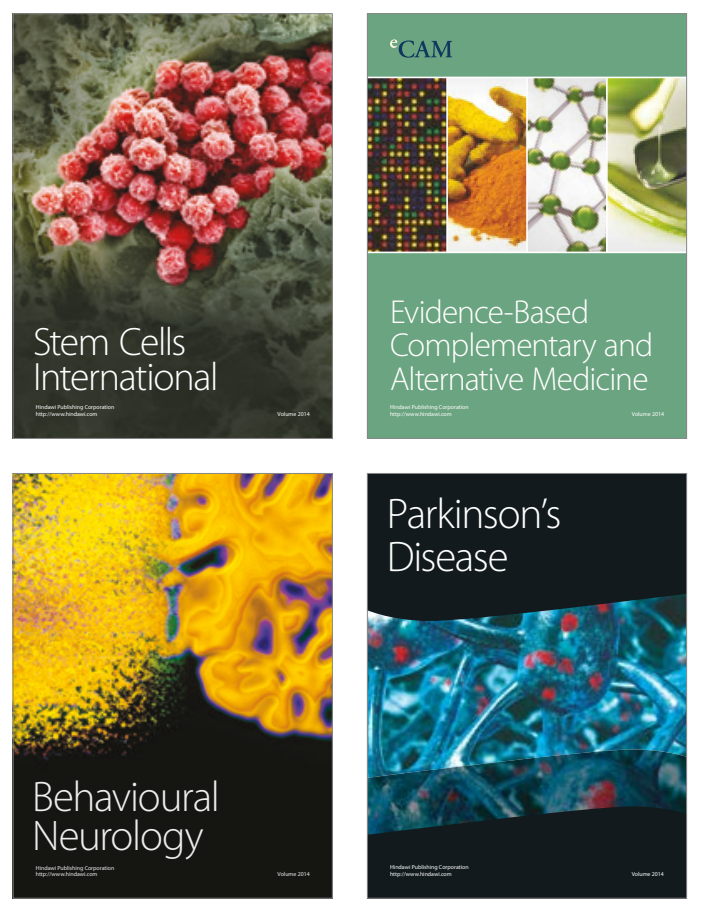
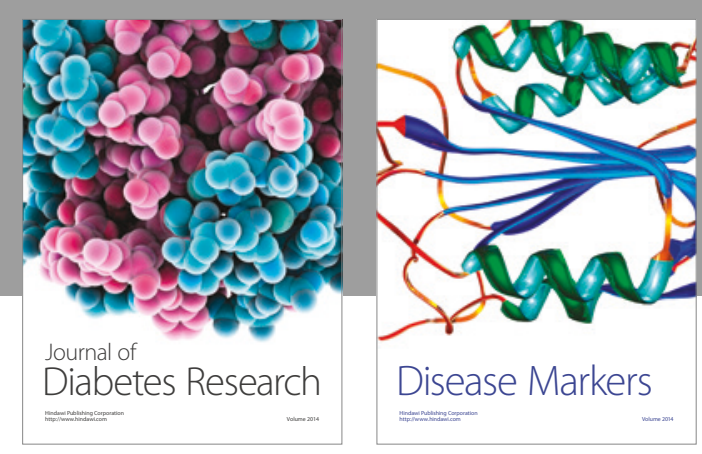

Disease Markers
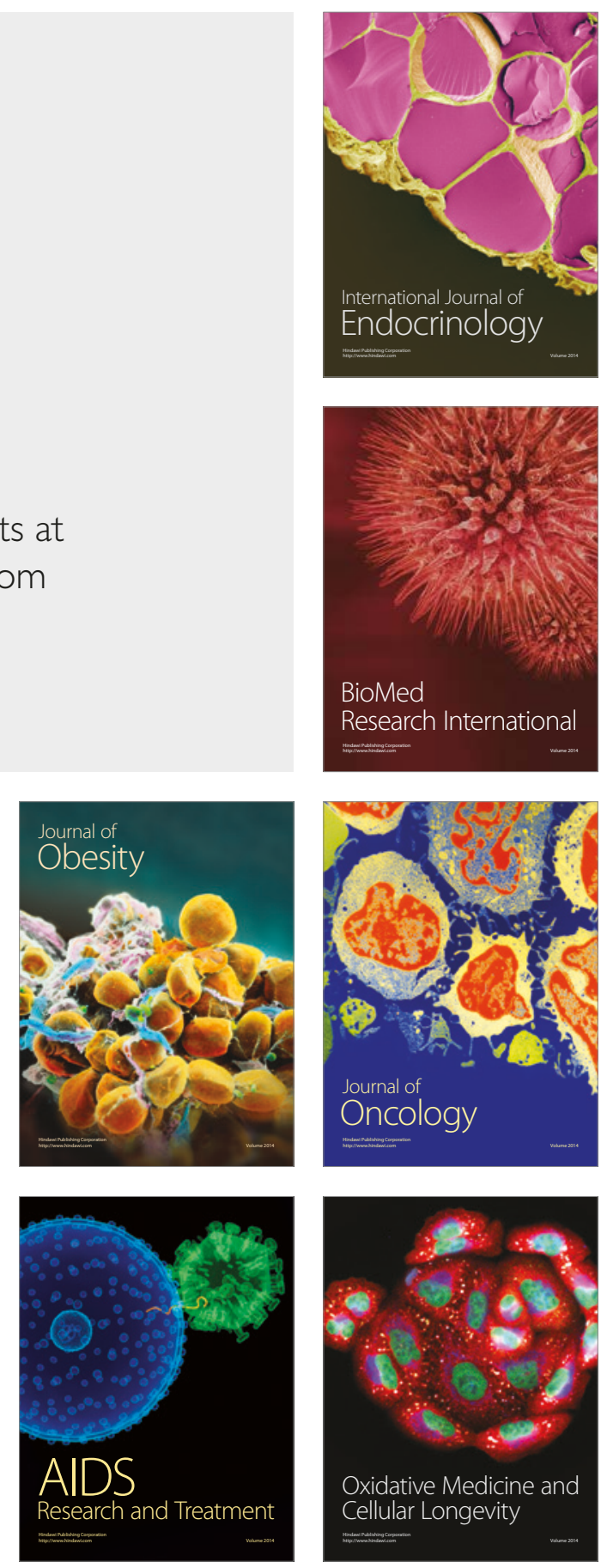